\title{
Performance Analysis of Massive Multi-input and Multi-output with Imperfect Channel State Information
}

\author{
Tasher Ali Sheikh*, Joyatri Bora, Anwar Hussain \\ Department of Electronics and Communication Engineering, North Eastern Regional Institute of Science and Technology, \\ Arunachal Pradesh 791109, India
}

Corresponding Author Email: tasher.ece@gmail.com

https://doi.org/10.18280/ts.360409

Received: 3 April 2019

Accepted: 18 July 2019

\section{Keywords:}

massive multi-input and multi-output (MIMO), 5G, user scheduling, antenna selection, scale fading, channel estimation error

\begin{abstract}
The type and state of the fading channel directly affect the performance of massive multi-input and multi-output (MIMO) system. For example, the small and large fading (SSF and LSF) of the channel have a great impact on the sum-rate of the system. However, the channel state information (CSI) is far from perfect, making it difficult to analyze the sum-rate of massive MIMO systems with uniform user distribution. To solve the problem, this paper proposes three scheduling algorithms, namely, semi-orthogonal user scheduling (SUS), random user scheduling (RUS), and distance-dependent user scheduling (DUS). The three algorithms were adopted to schedule different number of users $(8,10$ and 12), based on the maximum signalto-noise ratio (SNR) with changing number of base station antennas, number of active users, etc. The zero forcing (ZF) precoding was employed to improve the sum-rate, and the highly scattering Rayleigh fading channel was considered for both SSF and LSF, in the light of user locations. Under imperfect CSI and additional noise, the DUS achieved higher sum-rate than the other algorithms. The research results shed new light on the use of massive MIMO systems for $5 \mathrm{G}$ applications with high sum-rate requirements.
\end{abstract}

\section{INTRODUCTION}

Recently massive multiple input multiple output (MIMO) systems have drawn special attention in the cellular network, for data communication over conventional single input single output (SISO) systems. MIMO is seen as a key role player in terms of considerable improvement in the system capacity, spectrum efficiency, link reliability, energy efficiency, resistance to interference, providing quality of service, increase the coverage area, and degrees of freedom [1]. Massive MIMO also has appeared as the foundation and enabler element of the $5 \mathrm{G}$ cellular network for data communication. The increase in the capacity results from parallel transmissions to large number of users with a common spectrum. The base station (BS) contains a large number of antennas transmitting signals to a large number of users each with a single antenna or more than one antenna with different path that enhance capacity and link reliably of the system. Also, the system is able to resist intentional jamming and interference in such massive MIMO network. For the beamforming capacity with massive MIMO technology, the specified spectrum may be utilized [2]. The enhancement of system capacity and energy efficiency in massive MIMO system is totally dependent on joint user scheduling and base station antenna selection appropriately [3-6]. But joint selection of optimal number of best users and antennas in terms of some performance index of cellular massive MIMO network is not easy to achieve.

The strategy may require selecting a maximum number of users, each one allocated a base station antenna so that the system sum-rate is maximum possible. Also the system sumrate for transmitting data to a specified number of best users, each allocated one best antenna in some sense may be required to be maximized. Perfect and imperfect CSI is generally used in massive MIMO network for achieve maximum system sum rate derivation [7]. For user selection, mostly random user selection (RUS) and round robin algorithm (RRA) are widely used as it has very low computational cost for an approximate same capacity. As massive MIMO network has large number of users and antennas, the probability to obtain users with almost orthogonal channels is more. Hence semi-orthogonal user selection (SUS) is most popular and widely used method for very good system capacity and low interference and computational cost [8]. For efficient spectrum and energy efficiency, selections of the best users are required in conventional MIMO cellular network. In the same way it is also needed in the massive MIMO cellular network [9-12]. In conventional MIMO network the numbers of users are usually more than the numbers of base station antennas. But in massive MIMO network, numbers of base station antennas are very larger than the number of users. With $N$ number of base station antennas and $K$ number of users in the massive MIMO network, the cost of computational complexity become very large that is commonly $O\left(N^{3} K\right)$ for the SUS algorithm. The massive MIMO network may use both FDD and TDD frame work for user scheduling and data communication. The TDD based massive MIMO network is most popular as it allocates the whole spectrum to each individual user so that the system capacity improves significantly.

To obtain maximum system sum-rate and reduce computational cost within a limited backhaul capacity for downlink TDD based massive MIMO network, Xu et al. [13] proposed three joint antenna and semi-orthogonal user selection (JASUS) algorithms. In these algorithms, users 
contributing insignificantly to system sum-rate of massive MIMO network, where users are removed iteratively. A swapping based and simplified JASUS algorithm is suggested by Dong et al. [14] for downlink massive MIMO network with ZF precoding improving the system sum-rate and reducing the computational complexity. A low complexity JASUS algorithm with optimal system sum-rate growth is reported by Benmimoune et al. [15] where the users and antennas are removed step by step method. Moreover, to achieve higher spectral efficiency in massive MIMO non-orthogonal multiple access system, for single-band two-users and multi-band multi-users JASUS algorithm is presented by Liu and Wang [16]. For increase in the system sum-rate and minimization of feedback overhead for FDD based massive MIMO system, maximum channel gain based antenna group scheduling algorithm is suggested by Lee et al. [17].

In this paper we consider imperfect channel estimation by the base station from the pilot signal transmitted by the user antennas, each user has only one antenna for transmission and reception of signals. Hence there is channel estimation error which causes interference noise in the reception of the wanted signals. We also consider that the channel consists of both small scale fading (SSF) and large scale fading (LSF) components in its action on the transmitted signals. In such scenario of channel estimation error, we are interested to study performance of massive MIMO systems. We further consider that the users are distributed uniformly within a circular geographical area having a minimum distance from the base station which is not zero, equivalent to a real case scenario.

As we understand that TDD based massive MIMO system has great advantage, we consider the TDD frame work in this paper. For simultaneously serving the scheduled users by an equal number of base station antennas, we consider that the channel is estimated with pilot signal and linear simple zero forcing (ZF) precoding is used to diminish the interference. From a set of $X$ single antenna users and $M$ BS antenna, we select a pre-specified number of $K$ users and $N$ BS antennas, and maximize the system capacity, energy efficiency and spectral efficiency. For maximizing the system capacity, we consider simple three joint user scheduling and antenna selection algorithms: Distance Dependent User Scheduling and Maximum SNR based Antenna Selection (DUS-MSAS), Semi-orthogonal User Scheduling and Maximum SNR based Antenna Selection (SUS-MSAS), and Random User Scheduling and Maximum SNR based Antenna Selection (RUS-MSAS).

The contribution of this paper is summarized as: we have considered three algorithms such as DUS-MSAS, SUS-MSAS, and RUS-MSAS for data-rate improvement with ZF precoding. In DUS-MSAS algorithm, we selected those users nearest to the BS antenna and choose those base station antennas having maximum SNR with the selected users. In the SUS-MSAS, we selected the users those channel vectors are almost orthogonal with each other, and select those base station antennas having maximum SNR with the selected users. In RUS-MSAS algorithm, we selected the users randomly and choose those base station antennas having maximum SNR with the selected users. In this paper, we assumed that CSI is imperfect and introduces a term that may be considered as causing interference or additional noise.

The rest of the paper is organized as follows. The system model is presented in section-2, proposed algorithms are presented in section-3, results of simulation study are discussed in section-4, and conclusion of the work drawn is in section-5.

Notation: The Hermitian matrix, operation of conjugate transpose, pseudo-inversion denoted by $(\bullet)^{\perp}, \operatorname{tr}(\bullet)$ and $(\bullet)^{r}$ respectively. In this paper, the vector and matrix denoted as lower and upper case boldface letters respectively. The expected value with respect to the $\mathrm{x}$ is $E_{x}[\bullet]$. The symmetric complex Gaussian distribution for circular scenario is $C N(n, \lambda)$ where the mean vector is denoted as $n$ and covariance matrix is represented as $\lambda$. The absolute values of the scalar quantity of a vector, Frobenius norm of vector and transpose is denoted as, $|\cdot|,\|\cdot\|^{2}$ and $[\bullet]^{\mathrm{T}}$ respectively.

\section{SYSTEM MODEL}

We consider a downlink massive MIMO network that operates in TDD mode, the schematic diagram of which is shown in Figure 1. It consists of a single transmitter Base station equipped with $M$ antennas and $X$ single antenna receivers. The users are uniformly distributed in the cellular network excluding the shadow area as shown the figure. For each coherence time slot, the transmitter selects $K$ users from $X$ users and $N$ antennas from $M$ antennas of the BS for simultaneous data transmission, assuming that the magnitude of $N$ and $K$ are of same order. The channel SSF is assumed as Rayleigh distributed highly scattering and slowly fading over the block length of information. The LSF of the channel is due to signal fading of the users based on their distances from the BS.

From the $M$ antennas to $X$ users the composite channel matrix $\boldsymbol{C}$ for SSF and LSF characteristics is expressed as

$$
C=\mathbf{H} \sqrt{\mathrm{DR}}
$$

where, $\boldsymbol{C} \epsilon \mathbb{C}^{X \times M}$ and is the correlation matrix of transmit antennas which is modeled through $\delta$-Kac-Murdock-Szego matrix [18]. $\boldsymbol{H} \epsilon \mathbb{C}^{X \times M}$ is the SSF Rayleigh fading channel matrix with independent and identically distributed (i.i.d). Whose mean is zero and covariance is one. $\boldsymbol{D} \epsilon \mathbb{C}^{X \times M}$ is the main diagonal matrix and comprises of LSF coefficient $\beta_{k}$ and its value is $\beta_{k}=c d_{k}^{-a}$ where $k=1, \ldots, K, d_{k}$ is distance of the $\mathrm{k}^{\text {th }}$ user from BS, ' $a$ ' is the path loss exponent and ' $c$ ' is the reference LSF factor for wireless cellular network. We consider the channel to be uncorrelated and hence $\boldsymbol{R}=1$.

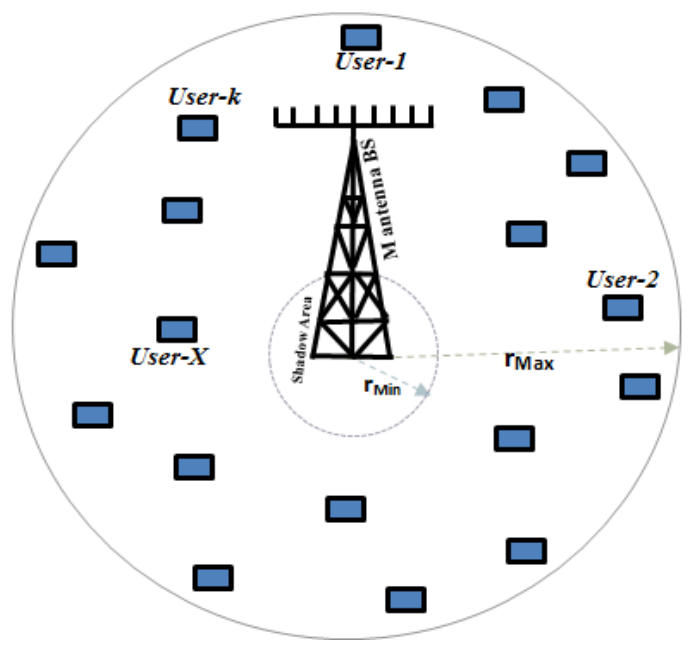

Figure 1. TDD based downlink massive MIMO model 
The BS achieves SSF CSI based on estimating the channel training signals as we assume the system operates in TDD mode. Hence SSF CSI matrix $\mathbf{H}$ is modeled as

$$
\mathbf{H}=\hat{\mathbf{H}}+\tilde{\mathbf{H}}
$$

where, the estimation channel matrix is $\hat{\boldsymbol{H}}=\left[\hat{\boldsymbol{h}}_{1}^{T}, \ldots, \hat{\boldsymbol{h}}_{K}^{T}\right]^{T}$ and the error channel matrix is $\widetilde{\boldsymbol{H}}=\left[\widetilde{\boldsymbol{h}}_{1}^{T}, \ldots, \widetilde{\boldsymbol{h}}_{K}^{T}\right]^{T}$ having dimension $K \times N$. The $\hat{\boldsymbol{H}}$ and $\widetilde{\boldsymbol{H}}$ can be written as

$$
\hat{\mathbf{H}}=\sqrt{1-\rho} \mathbf{U}_{1} ; \quad \tilde{\mathbf{H}}=\sqrt{\rho} \mathbf{U}_{2}
$$

where, $0 \leq \rho \leq 1$ and the $k^{\text {th }}$ row vectors of $\hat{\boldsymbol{H}}$ and $\widetilde{\boldsymbol{H}}$ are $\hat{\boldsymbol{h}} \varepsilon \mathbb{C}^{1 \times N}$ and $\widetilde{\boldsymbol{h}} \varepsilon \mathbb{C}^{1 \times N}$ respectively for the $k^{\text {th }}$ user, with $\boldsymbol{U}_{1}$ and $\boldsymbol{U}_{2}$ as two independent matrices modeled as i.i.d $C N(0,1)$. In our model we consider that at the BS, LSF CSI is perfectly known for selected users and antennas. Therefore, from Eq. (2) and Eq. (3), the Eq. (1) can be rewritten for downlink channel matrix as

$$
\mathbf{C}=\hat{\mathbf{C}}+\tilde{\mathbf{C}}
$$

Using Eq. (2) in the above equation we have

$$
\begin{aligned}
& \mathbf{C}=(\hat{\mathbf{H}}+\tilde{\mathbf{H}}) \sqrt{\mathbf{D R}} \\
& \mathbf{C}=\hat{\mathbf{H}} \sqrt{1 . c d_{k}^{-a}}+\tilde{\mathbf{H}} \sqrt{1 . c d_{k}^{-a}} \\
& \mathbf{C}=\hat{\mathbf{H}} \sqrt{c d_{k}^{-a}}+\tilde{\mathbf{H}} \sqrt{c d_{k}^{-a}}
\end{aligned}
$$

Considering ZF linear precoding scheme to nullify the interference amongst the selected users due to imperfect SSF CSI Rayleigh fading channel, the transmit vector $\mathbf{x} \varepsilon \mathbb{C}^{N \times 1}$, is written as

$$
\begin{gathered}
\mathbf{x}=\varsigma \hat{\mathbf{C}}^{\Upsilon} s \\
\mathbf{x}=\varsigma \hat{\mathbf{C}}^{\perp}\left(\hat{\mathbf{C}} \hat{\mathbf{C}}^{\perp}\right)^{-1} s
\end{gathered}
$$

where, $\hat{\boldsymbol{C}}^{r}$ is the pseudo-inverse vector of $\hat{\boldsymbol{C}}$ and $\varsigma$ is the power controlling factor. The data bearing transmit symbol vector is $\bar{s}=\left[s_{1}, \ldots, s_{K}\right]^{T}$ and $k^{\text {th }}$ selected users symbol is represented as $S_{k}$. Assuming $P$ as total system power, the constraint of long-term power is as bellow.

$$
P \geq E\left[\operatorname{tr}\left(\mathbf{x x}^{\perp}\right)\right]
$$

The power controlling factor $\varsigma$ can be calculated as in Eq.

$$
\varsigma=\sqrt{\frac{P}{\operatorname{tr}\left(\hat{\mathbf{C}} \hat{\mathbf{C}}^{\perp}\right)^{-1}}}
$$

To reduce the system computational cost in our proposed model, we assume equal power allocation to each selected users. Two vectors $\boldsymbol{u}$ and $\boldsymbol{v}$ are said to be $\vartheta$-orthogonal if $\vartheta \leq 0$ [19] which is mathematically represented as-

$$
\vartheta \geq \frac{\left|\mathbf{u}^{\perp} \mathbf{v}\right|}{\|\mathbf{u}\|\|\mathbf{v}\|}
$$

where, the value of $\vartheta$ is positive as shown in Ref. [20]. With the aid of Eq. (7) the received signal vector of $K \times 1$ dimension is given as-

$$
\mathbf{r}=\mathbf{C x}+\boldsymbol{\omega}
$$

Utilizing Eq. (4) in Eq. (10) we have

$$
\mathbf{r}=(\hat{\mathbf{C}}+\tilde{\mathbf{C}}) \mathbf{x}+\omega
$$

Using Eq. (6) and Eq. (7) in the above equation, it can be rewritten as-

$$
\begin{gathered}
\mathbf{r}=\varsigma(\hat{\mathbf{C}}+\tilde{\mathbf{C}}) \hat{\mathbf{C}}^{\Upsilon} s+\boldsymbol{\omega} \\
\mathbf{r}=\varsigma \hat{\mathbf{C}} \hat{\mathbf{C}}^{\gamma} s+\varsigma \tilde{\mathbf{C}} \hat{\mathbf{C}}^{\Upsilon} s+\boldsymbol{\omega} \\
\mathbf{r}=\varsigma \hat{\mathbf{C}} \hat{\mathbf{C}}^{\perp}\left(\hat{\mathbf{C}} \hat{\mathbf{C}}^{\perp}\right)^{-1} s+\varsigma \tilde{\mathbf{C}} \hat{\mathbf{C}}^{r} s+\boldsymbol{\omega} \\
\mathbf{r}=\varsigma s+\varsigma \tilde{\mathbf{C}} \hat{\mathbf{C}}^{\gamma} s+\boldsymbol{\omega}
\end{gathered}
$$

In Eq. (11), $\boldsymbol{\omega}=\left[\boldsymbol{\omega}_{1}, \ldots, \boldsymbol{\omega}_{\mathrm{k}}\right]^{\mathrm{T}} \boldsymbol{\epsilon} \mathbb{C}^{\mathrm{K} \times 1}$ is the additive white Gaussian noise vector and $\omega_{k} \approx C N\left(0, \sigma_{n}^{2}\right)$, at the receiver user terminal. The received signal vector for the $k^{\text {th }}$ selected user is given by

$$
r_{k}=\varsigma s_{k}+\varsigma \sum_{k=N, j \neq k} \tilde{\mathbf{C}}_{k} \hat{\mathbf{C}}_{j}^{\curlyvee} s_{j}+\boldsymbol{\omega}_{k}
$$

Using Eq. (5) in Eq. (12), the above can be rewritten as

$$
r_{k} \cong \varsigma s_{k}+\varsigma \sum_{k=N, j \neq k} \sqrt{c d_{k}^{-a}} \tilde{\mathbf{h}}_{\mathbf{k}} \hat{\mathbf{C}}_{j}^{\Upsilon} s_{j}+\boldsymbol{\omega}_{k}
$$

where, $\widetilde{\boldsymbol{h}}$ is $k^{\text {th }}$ row vector of $\widetilde{\boldsymbol{H}}$. The first term of Eq. (13) is the desired signal power of the data symbol. The second term of Eq. (13) may be considered as an additional noise term over the normal Gaussian noise and which is considered here as the interference term arising out because of imperfect channel estimation. Hence, we rewrite the Eq. (13) as

$$
r_{k} \cong \varsigma s_{k}+\varsigma \sum_{k=N, j \neq k} \sqrt{c d_{k}^{-a}} \mathbf{h}_{\mathbf{k}} \mathbf{C}_{\mathbf{j}}^{\Upsilon} s_{j}+\boldsymbol{\omega}_{k}
$$

The equation of SINR for $k^{\text {th }}$ the receiver user can be written as

$$
\mathbf{S I N R}_{\mathbf{k}}=\frac{\varsigma^{2}}{\sigma_{n}^{2}+\varsigma^{2} \sum_{k=N, j \neq k} c d_{k}^{-a}\left|\mathbf{h}_{\mathbf{k}} \mathbf{C}_{\mathbf{j}}^{\gamma}\right|^{2}}
$$

The system sum-rate of selected users and selected antennas is given by

$$
\mathfrak{R}=\Phi \sum_{k=1}^{N} \log _{2}\left(1+\mathbf{S I N R}_{\mathbf{k}}\right)
$$


where, $\Phi=\{1-(N$ or $K / \tau)\}$ is the Prelog factor and $\tau$ is the coherence time in the considered slot and is constant.

\section{PROPOSED ALGORITHMS}

In this paper, as stated earlier, we study performance of massive MIMO systems using the three algorithms, namely DUS-MSAS (Algorithm- $A$ ), SUS-MSAS (Algorithm- $B$ ) and RUS-MSAS (Algorithm- $C$ ). In algorithm- $A$, users nearest to the BS are scheduled, in algorithm- $B$, users that are mutually orthogonal or semi-orthogonal to each other, are scheduled, and in algorithm- $C$, users are scheduled randomly. In all the algorithms the base station antennas are selected based on maximum SNR to the scheduled receivers. Algorithms $A, B, C$ are expressed by the following.

\section{Algorithm A: Distance Dependent User Scheduling and Maximum SNR based Antenna Selection (DUS-MSAS).}

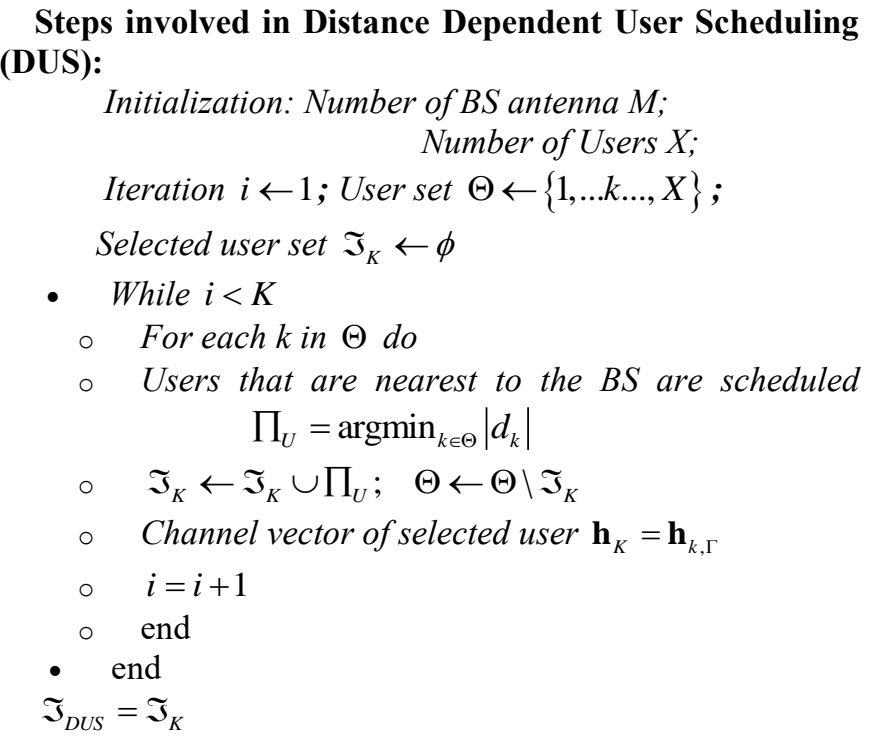

\section{Steps involved in Antenna Selection:}

For each user in $\mathfrak{I}_{\text {DUS }}$, Channel vector of selected user $\mathbf{h}_{K}=\mathbf{h}_{k, \Gamma}$

No. of BS antenna $M$; Iteration $j \leftarrow 1$;

Base station set $\Gamma \leftarrow\{1, \ldots m, \ldots, M\}$;

Set of selected base station antenna $\mathfrak{I}_{K, N} \leftarrow \phi$

- While $j \leq N$

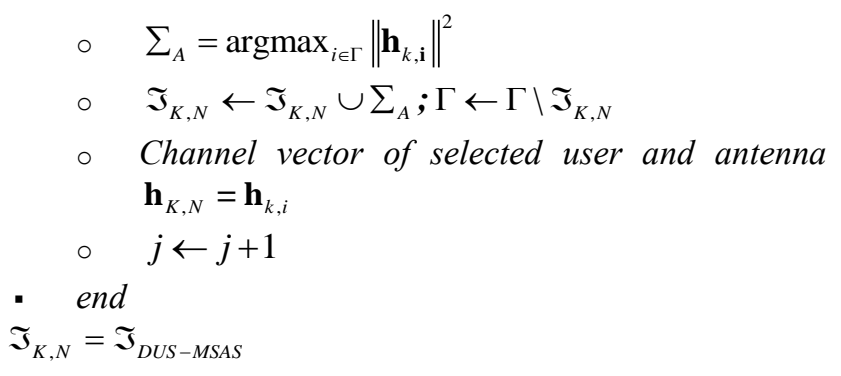

\footnotetext{
Algorithm-B: Semi-orthogonal User Scheduling and Maximum SNR based Antenna Selection (SUS-MSAS).
}

Steps involved in Semi-orthogonal User Scheduling;

Initialization: Number of BS antenna $M$; Number of User $X$;
Iteration $i \leftarrow 1$; User set $\Theta \leftarrow\{1, \ldots k \ldots, X\}$;

Selected user set $\mathfrak{I}_{K} \leftarrow \phi$

To select first reference user, $\mathfrak{\Im}_{1}=\operatorname{argmax}_{\kappa \in \Theta}\left\|\mathbf{h}_{\mathbf{k}, \Gamma}\right\|^{2}$

$\mathfrak{I}_{K} \leftarrow \mathfrak{I}_{K} \cup \mathfrak{I}_{1}$

- While $i<K$

- For each $k$ in $\Theta$ do

$\circ \frac{\left|\mathbf{h}_{\kappa}^{\perp} \mathbf{h}_{\mathrm{k}-\mathbf{1}}\right|}{\left\|\mathbf{h}_{\mathrm{k}}\right\|\left\|\mathbf{h}_{\mathrm{k}-\mathbf{1}}\right\|} \leq \vartheta$

$\circ \quad$ end

○ $\prod_{U}=\operatorname{argmax}_{k \in \Theta}\left\|\mathbf{h}_{\mathbf{k}, \Gamma}\right\|^{2}$

○ $\mathfrak{I}_{K} \leftarrow \mathfrak{I}_{K} \cup \prod_{U} ; \quad \Theta \leftarrow \Theta \backslash \mathfrak{I}_{K}$

- Channel vector of selected user $\mathbf{h}_{K}=\mathbf{h}_{k, \Gamma}$

- end $i=i+1$

$\mathfrak{I}_{S U S}=\mathfrak{I}_{K}$

For each user in $\mathfrak{I}_{\text {SUS }}$

Steps involved in Antenna Selection:

Same as in Algorithm- $A$

$\mathfrak{I}_{K, N}=\mathfrak{I}_{S \mathrm{U} S-M S A S}$

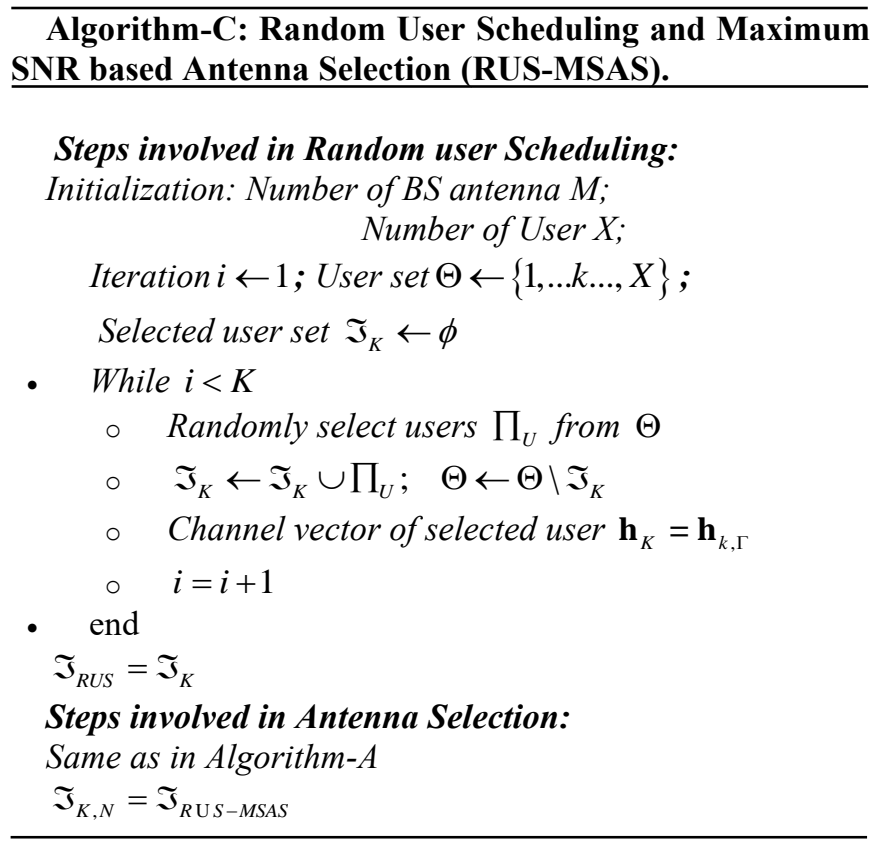

\section{RESULTS AND DISCUSSION}

For exploring the performance of DUS-MSAS, SUS-MSAS and RUS-MSAS algorithms with ZF precoding, we experiment with simulation of the massive MIMO transmissions varying the SNRs, the number of BS antennas, and the number of users. Throughout the simulation we consider different parameters with values as shown in Table 1. The simulation setting is done in Matlab.

Figure 2 shows the performance of massive MIMO with configurations of $8 \times 8,10 \times 10$ and $12 \times 12$ sets of selected users and selected base station antennas with a variation of SNR, and with $M=64$ and $X=32$. The simulation results as shown in Figure 2 (A), Figure 2 (B), and Figure 2 (C), show that the average sum-rate of the system increases linearly with SNR. Out of the three proposed algorithms DUS-MSAS has the 
highest rate whereas RUS-MSAS has the lowest. The DUSMSAS and SUS-MSAS have almost the same system sum-rate. At lower SNR the difference of sum-rate between algorithms is very low and at high SNR the RUS-MSAS lags significantly from the other two.

Table 1. Simulation component value

\begin{tabular}{cccc}
\hline Parameters & Value & Parameters & Value \\
\hline$N=K$ & $8 / 10 / 12$ & $R_{\min }$ & $75 \mathrm{~m}$ \\
\hline$\delta$ & 1 & $P$ & $25 \mathrm{dBm}$ \\
\hline$a$ & 3 & $M$ & 64 \\
\hline$c$ & $10^{-3.53}$ & $X$ & 32 \\
\hline$\sigma_{n}{ }^{2}$ & $-96 \mathrm{dBm}$ & $\vartheta$ & 0.1 \\
\hline$\tau$ & 128 & $B W$ & $3.5 \mathrm{GHz}$ \\
\hline$R_{\max }$ & $350 m$ & & \\
\hline
\end{tabular}
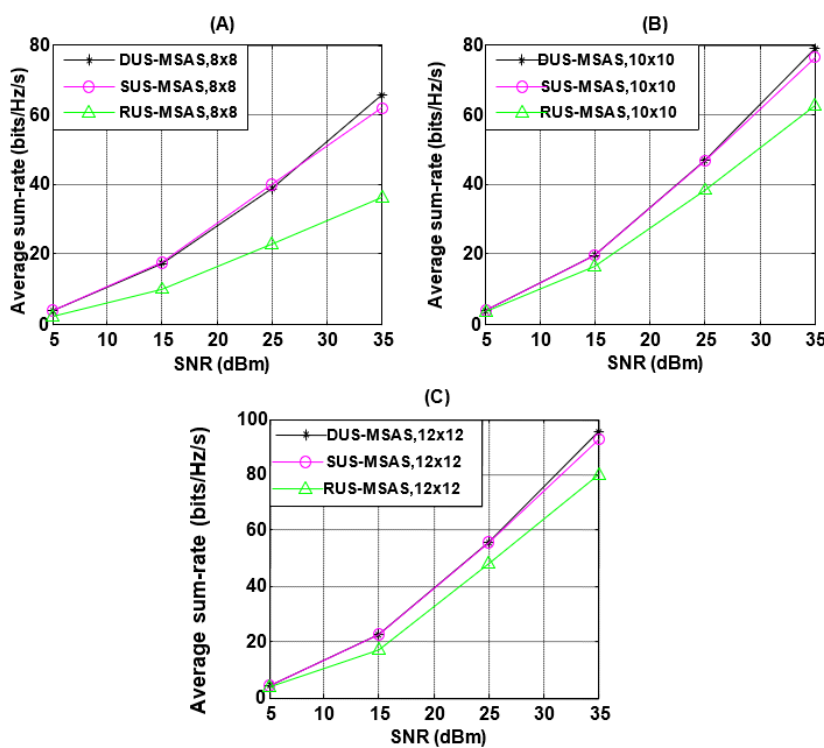

Figure 2. Average system sum-rate vs. SNR performance of DUS-MSAS, SUS-MSAS, and RUS-MSAS algorithm for (A) $8 \times 8$, (B) $10 \times 10$, and (C) $12 \times 12$ configuration, with $M$ $=64$ and $X=32$

(A)
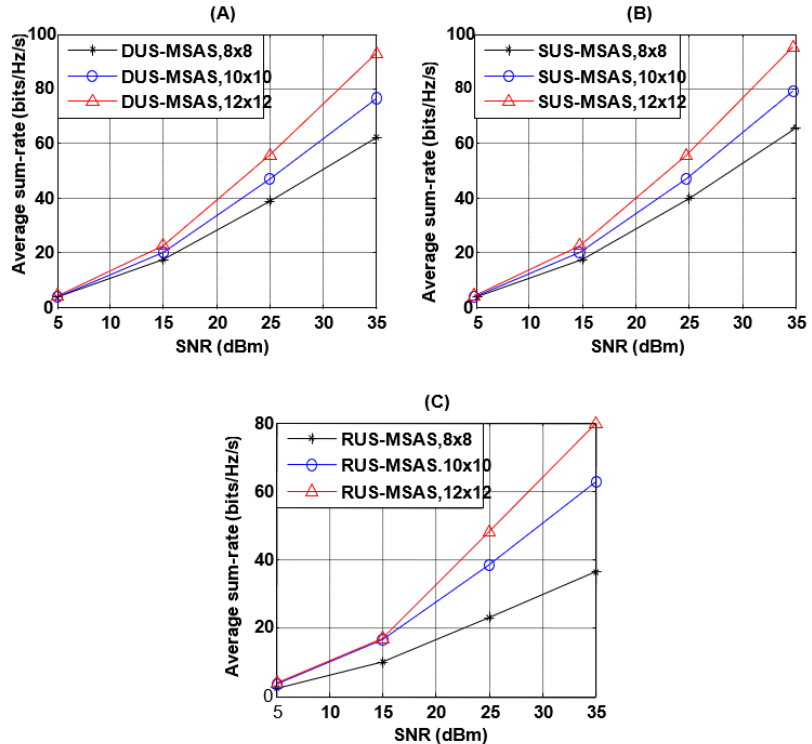

Figure 3. Average system sum-rate vs. SNR for $8 \times 8,10 \times 10$ and $12 \times 12$ configuration, (A) DUS-MSAS (B) SUS-MSAS

(C) RUS-MSAS, with $M=64$ and $X=32$
We now plot the performance of each algorithm separately with all the three configurations as shown in Figure 3 keeping $M$ and $X$ same. It is observed from Figure 3 that the average sum-rate increases with a greater number of users and antennas in the configuration. It is also clear from Figure 3 (A), Figure $3(\mathrm{~B})$, and Figure $3(\mathrm{C})$ that the average sum-rate increases almost linearly with SNR and at lower SNR the difference of rates between configurations is small and at higher SNR the difference in rates is higher. For all the three $8 \times 8,10 \times 10$, and $12 \times 12$ configurations, DUS-MSAS and SUS-MSAS perform almost equally.
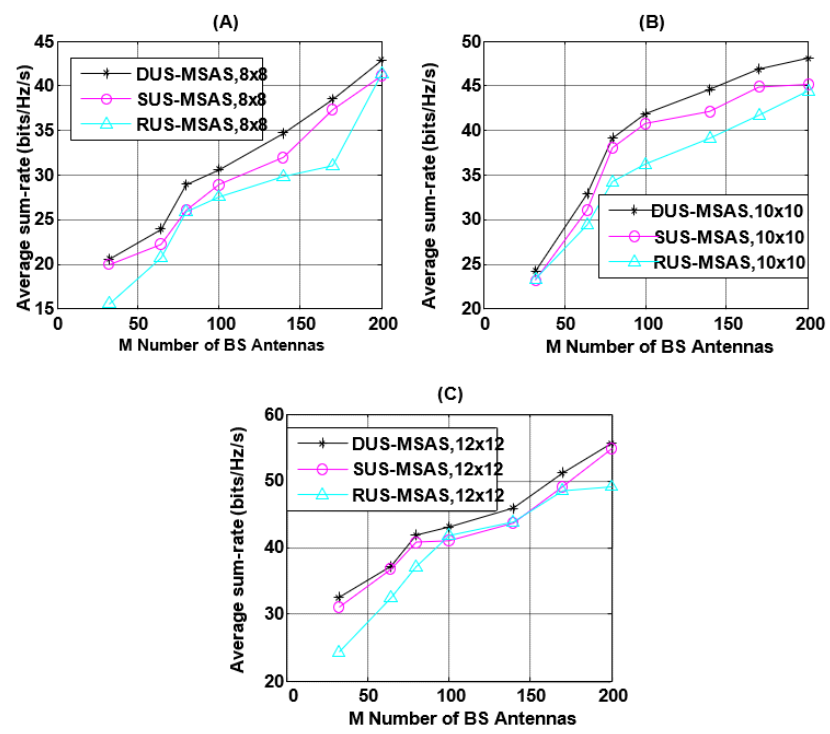

Figure 4. Average system sum-rate vs. M of DUS-MSAS, SUS-MSAS, and RUS-MSAS algorithms, (A) $8 \times 8$, (B)

$10 \times 10$, and $(\mathrm{C}) 12 \times 12$ configuration, with constant $S N R=25 \mathrm{dBm}$ and $X=64$
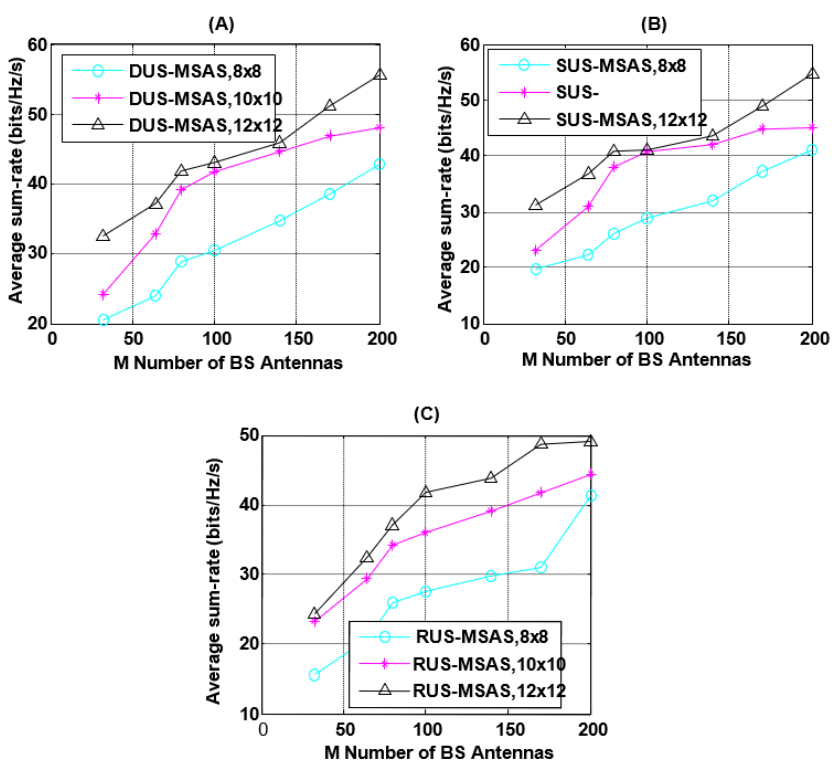

Figure 5. Average sum-rate vs. $M$ for $8 \times 8,10 \times 10$ and $12 \times 12$ configuration, (A) DUS-MSAS, (B) SUS-MSAS (C) RUSMSAS, with constant $S N R=25 \mathrm{dBm}$ and $X=64$

We now explore the effect of variation of $M$ on the system sum rate for a specific $S N R$ and $X$. Varying $M$ from 45 to 200, for fixed $X=64$ and $S N R=25 \mathrm{dBm}$, the system sum-rate performance is presented in Figure 4 . For all the three 
algorithms, the system sum-rate for $8 \times 8,10 \times 10$ and $12 \times 12$ configurations of sets of selected users and antennas are investigated. As is shown in the figures, the average system sum-rate rises non-linearly with the variation of $M$. It is also noticed in Figure 4 that for all configurations the DUS-MSAS has higher average system sum-rate than SUS-MSAS and RUS-MSAS, although the difference between the first two is small. We also plot the results for each algorithm separately and are shown in Figure 5. From Figure 5 (A), (B), and (C), we comment that the average system sum-rate increases in every algorithm with increase in the size of the configuration and the trend is more or less same amongst different algorithms.

In Figure 6 and Figure 7 we show the performance of the massive MIMO system with $M=64, S N R=25 \mathrm{dBm}$ for multiuser diversity with varying number of active users $X$ and for all algorithms with $8 \times 8,10 \times 10$ and $12 \times 12$ configurations. We observe from Figure 6 that DUS-MSAS provide highest average system sum-rate in all configurations. Also the trend of increase of average sum-rate with variation of $X$ is almost similar in each configuration for all the algorithms. In Figure 7 we show the performance for each algorithm separately for all the three configurations with varying $X$. We observe that there is significant increase of sum-rate with rise of configuration sizes.
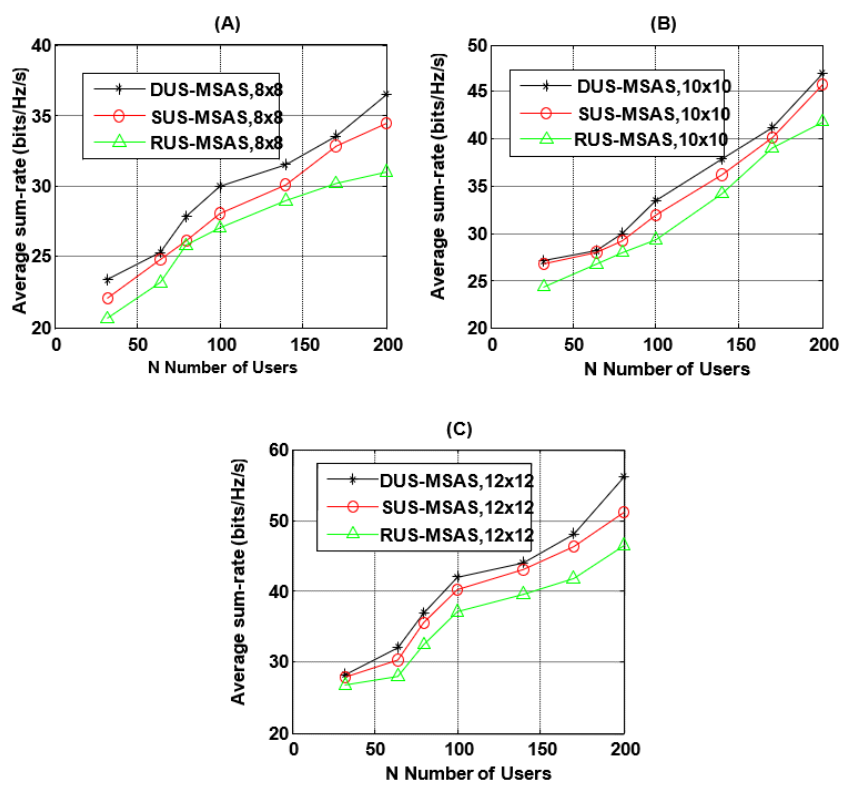

Figure 6. Average sum-rate vs. $N$ for DUS-MSAS, SUSMSAS, and RUS-MSAS, (A) $8 \times 8$, (B) $10 \times 10$, (C) $12 \times 12$ configuration, with fixed $M=64$ and $S N R=25 \mathrm{dBm}$

Throughout this paper we have achieved highest average system sum-rate using DUS-MSAS algorithm. In this algorithm users are chosen based on distance knowledge, i.e. those users are scheduled that are nearest to the BS. This indicates that nearest users are favored and distant users suffered. On the other hand, in the SUS-MSAS, users having almost orthogonal channel are scheduled, although it draws effect from distance also. In RUS-MSAS algorithm users are chosen randomly and hence we observe sum-rate is not encouraging. For DUS-MSAS it is required that BS has distance information of all the users, whereas in SUS-MSAS BS obtains CSI based on pilot signals from users and channel having same gain in forward and reverse directions over the information length. Also it is observed that the performance difference between DUS-MSAS and SUS-MSAS is very small. We have left the delay incurred in the computation of each algorithm for our future work. However, with intense computational facility with the base station, the delay may be expected to be within limits.
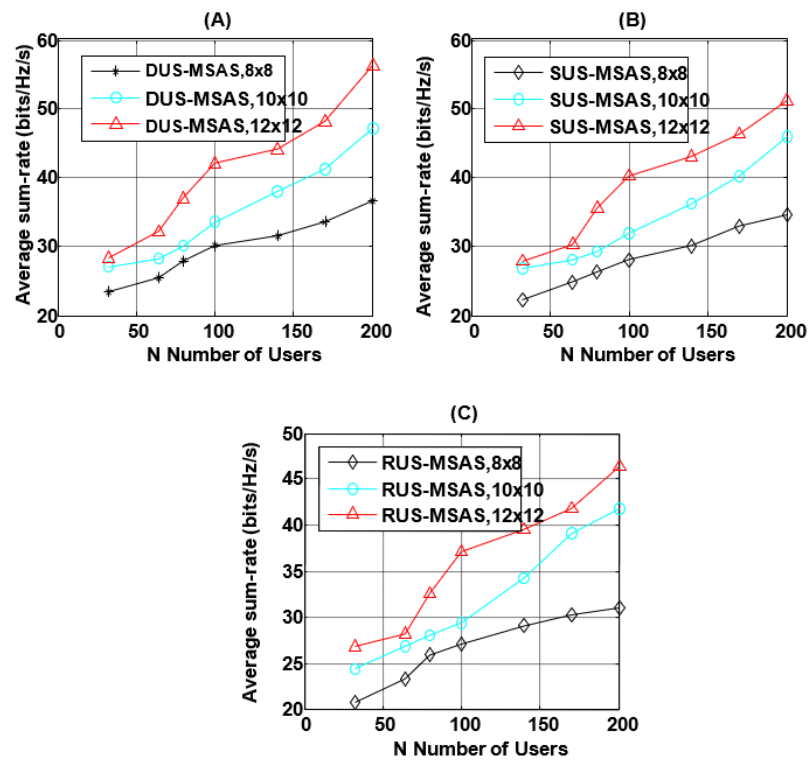

Figure 7. Average sum-rate vs. $N$ for $8 \times 8,10 \times 10$ and $12 \times 12$ configuration, (A) DUS-MSAS, (B) SUS-MSAS, (C)-of RUS-MSAS, with fixed $M=64$ and $S N R=25 \mathrm{dBm}$

\section{CONCLUSIONS}

We show simulation results for performance of massive MIMO system, assuming imperfect CSI and considering both SSF and LSF with ZF precoding, with variation in the number of base station antennas, number of active users, and SNR. The evaluation of performance is studied for three user scheduling techniques SUS, RUS, DUS, and antenna selection based on maximum SNR in all cases. The users are distributed uniformly in the geographical area of radius 350 meters. The results show that the average system sum-rate increases nonlinearly with number of BS antennas irrespective of scheduling technique, DUS-MSAS being the highest. The variation of average system sum rate with number of active users, as found from results shows that the system capacity increases with number of active users. We also studied performance variation of the system with SNR and the results show that DUS-MSAS and SUS-MSAS provide almost equal average system sum rate, RUS-MSAS being the lowest. The performance for $8 \times 8,10 \times 10$, and $12 \times 12$ increases with increase in SNR, higher number of users having more increase in the sum-rate. In this study we have not considered the cost of implementation in terms of number of bits for each scheduling technique, the further analysis of which we intend to report in our future publication.

\section{ACKNOWLEDGMENT}

We acknowledge hereby the financially support of the Ministry of Electronics and IT (Meity), Government of India, under the project of Visvesvaraya $\mathrm{PhD}$ scheme, for this research (Grant no.: PhD-MLA-4(96)/2015-2016). 


\section{REFERENCES}

[1] Larsson, E.G., Edfors, O., Tufvesson, F., Marzetta, T.L. (2014). Massive MIMO for next generation wireless system. IEEE Communications Magazine, 52(2): 186195. https://doi.org/10.1109/MCOM.2014.6736761

[2] Sheikh, T.A., Bora, J., Hussain, M.A. (2017). A survey of antenna and user scheduling techniques for massive MIMO-5G wireless system. 2017 International Conference on Current Trends in Computer, Electrical, Electronics and Communication (CTCEEC), pp. 578583. https://doi.org/10.1109/CTCEEC.2017.8455177

[3] Sheikh, T.A., Bora, J., Hussain, M.A. (2019). Combined user and antenna selection in massive MIMO using precoding technique. International Journal of Sensors, Wireless Communications and Control, 9(2): 214-223. https://doi.org/10.2174/2210327908666181112144939

[4] Sheikh, T.A., Bora, J., Hussain, M.A. (2018). Sum-rate performance of massive MIMO systems in highly scattering channel with semi-orthogonal and random user selections. Radioelectronics and Communications Systems, 61(12): 547-555. https://doi.org/10.3103/S0735272718120026

[5] Sheikh, T.A., Bora, J., Hussain, M.A. (2019). Capacity Maximizing in massive MIMO with linear precoding for SSF and LSF channel with perfect CSI. Digital Communications and Networks. https://doi.org/10.1016/j.dcan.2019.08.002

[6] Sachan, V., Kumar, I., Shankar, R., Mishra, R.K. (2018). Analysis of transmit antenna selection based selective decode forward cooperative communication protocol. $\begin{array}{llll}\text { Traitement } & \mathrm{du} & \text { Signal, } & \text { 35(1): }\end{array}$ https://doi.org/10.3166/TS.35.47-60

[7] Sachan, V., Mishra, R.K. (2019). Uplink sum rate and capacity of hybrid precoding mmWave massive MIMO system. Traitement du Signal, 36(2): 155-160. https://doi.org/10.18280/ts.360205

[8] Liu, H., Gao, H., Yang, S., Lv, T. (2017). Lowcomplexity downlink user selection for massive MIMO system. IEEE System Journal, 11(2): 1072-1083. https://doi.org/10.1109/JSYST.2015.2422475

[9] Bjornson, E., Sanguinetti, L., Hoydis, J., Debbah, M. (2015). Optimal design of energy-efficient multi-user MIMO system: Is massive MIMO the answer? IEEE Transactions on Wireless Communications, 14(6): 30593075. https://doi.org/ 10.1109/TWC.2015.2400437

[10] Nam, J., Adhikary, A., Ahn, J.Y., Caire, G. (2014). Joint spatial division and multiplexing: Opportunistic beamforming, user grouping and simplified downlink scheduling. IEEE Journal of Selected Topics in Signal Processing, 8(5): 876-890. https://doi.org/10.1109/JSTSP.2014.2313808

[11] Lee, G., Sung, Y. (2014). Asymptotically optimal simple user scheduling for massive MIMO downlink with twostage beamforming. 2014 IEEE 15th International Workshop on Signal Processing Advances in Wireless Communications (SPAWC), pp. 60-64. https://doi.org/10.1109/SPAWC.2014.6941317

[12] Xu, Y., Yue, G., Mao, S. (2014). User grouping for massive MIMO in FDD system: New design methods and analysis. IEEE Access, 2: 947-959. https://doi.org/10.1109/ACCESS.2014.2353297

[13] Xu, G., Liu, A., Jiang, W., Xiang, H., Luo, W. (2014). Joint user scheduling and antenna selection in distributed

massive MIMO systems with limited backhaul capacity. China Comunications, 11(5): 17-30. https://doi.org/10.1109/CC.2014.6880457

[14] Dong, Y., Tang, Y., Zhang, K. (2017). Improved joint antenna selection and user scheduling for massive MIMO systems. 2017 IEEE/ACIS 16th International Conference on Computer and Information Science (ICIS), pp. 69-74. https://doi.org/ 10.1109/ICIS.2017.7959971

[15] Benmimoune, M., Driouch, E., Ajib, W., Massicotte, D. (2015). Joint transmit antenna selection and user scheduling for Massive MIMO systems. IEEE Wireless Communications and Networking Conference (WCNC), New Orleans, LA, pp. 381-386. https://doi.org/10.1109/WCNC.2015.7127500

[16] Liu, X., Wang, X. (2016). Efficient antenna selection and user scheduling in $5 \mathrm{G}$ massive MIMO-NOMA system. IEEE 83rd Vehicular Technology Conference (VTC Spring), pp. 1-5. https://doi.org/10.1109/VTCSpring.2016.7504208

[17] Lee, B., Ngo, L., Shim, B. (2014). Antenna group selection based user scheduling for massive MIMO systems. IEEE Global Communications Conference, Austin, TX, 3302-3307. https://doi.org/10.1109/GLOCOM.2014.7037316

[18] Huh, H. (2012). Large system analysis of multi-cell MIMO downlink: Fairness scheduling and inter-cell cooperation. Ph.D. dissertation, University of Southern California, Los Angeles, CA, USA.

[19] Yoo, T., Goldsmith, A. (2006). On the optimality of multiantenna broadcast scheduling using zero-forcing beamforming. IEEE Journal on Selected Areas in Communication, 24(3): 528-541. https://doi.org/10.1109/JSAC.2005.862421

[20] Zelst, A.V., Hammerschmidt, J.S. (2002). A single coefficient spatial correlation model for multiple-input multiple-output (MIMO) radio channels. 27 $7^{\text {th }}$ General Assembly of the Intl. Union of Radio Science (URSI), Maastricht, Netherlands, pp. 1-4.

\section{NOMENCLATURE}

M

$\mathrm{X}$

$\mathrm{K}$

$\mathrm{N}$

C

H

D

$\mathrm{d}_{\mathrm{k}}$

a

$\mathbf{R}$

$\hat{\mathbf{H}}$

$\widetilde{\boldsymbol{H}}$

$\mathbf{x}$

S

$\mathrm{P}$

r

\section{Greek symbols}

$\begin{array}{ll}(\bullet)^{\perp} & \text { Hermitian matrix } \\ \operatorname{tr}(\bullet) & \text { Conjugate transpose }\end{array}$

Total Number of Base Station Antenna
Total Number of Users
Selected Users
Selected Base Station Antenna
composite channel matrix
Channel matrix
diagonal matrix
distances between user to BS
path loss exponent
reference LSF factor
correlation matrix
estimation channel matrix
error channel matrix
transmit vector
data bearing transmit signal
total transmit power
radius (meter)

Total Number of Base Station Antenna Selected Users composite channel matrix Channel matrix path loss exponent matrix transmit vector total transmit power

(1)


Pseudo-inversion

Expected value with respect to $\mathrm{x}$

Complex gussain distribution with $n$ mean and variance $\lambda$

Absulate value

Frobenius norm

transpose

LSF coefficient

power controlling factor

Angle between two channel vector $\omega$

$\sigma_{n}^{2}$

$\phi$

$\tau$

\section{Subscripts}

$\mathrm{dBm} \quad$ decibel-milliwatts

$\mathrm{m}$ meter

$\mathrm{Hz} \quad \mathrm{Hertz}$
Additive white Gaussian noise covariance value

Prelog factor

Coherence time 